Annals of Pure and Applied Mathematics

Vol. 14, No. 3, 2017, 379-386

ISSN: 2279-087X (P), 2279-0888(online)

Published on 11 October 2017

www.researchmathsci.org

DOI: http://dx.doi.org/10.22457/apam.v14n3a4

Annals of

Pure and Applied

Mathematics

\title{
Prime Cordial Labeling for Theta Graph
}

\author{
A. Sugumaran ${ }^{1}$ and P.Vishnu Prakash ${ }^{2}$ \\ Department of Mathematics, Government Arts College \\ Tiruvannamalai, Tamilnadu, India. \\ ${ }^{1}$ Email:sugumaran3@yahoo.com \\ ${ }^{2}$ Corresponding author. Email: vishnuprakashp78@gmail.com
}

Received 20 July 2017; accepted 1 August 2017

Abstract. A prime cordial labeling of a graph $G=(V(G), E(G))$ is a bijection $f$ from $\mathrm{V}(\mathrm{G})$ to $\{1,2, \ldots|V(G)|\}$ such that for each edge $u v$ is assigned the label 1 if $\operatorname{gcd}(f(u), f(v))=1$ and 0 if $\operatorname{gcd}(f(u), f(v))>1$; then the number of edges labeled with 0 and the number of edges labeled with 1 differ by at most 1 . A graph which admits a prime cordial labeling is called a prime cordial graph. In this paper, we investigate the prime cordial labeling of Theta graph. We also discuss prime cordial labeling in the context of some graph operations namely duplication, switching, fusion, path union and the graph obtained by joining two copies of Theta graph by a path of arbitrary length .

Keywords: Prime cordial labeling, fusion, switching, contraction, path union.

\section{AMS Mathematics Subject Classification (2010): $05 \mathrm{C} 78$}

\section{Introduction}

Labeling of vertices and edges play a vital role in graph theory. Graph labeling is an active area of research in graph theory which has rigorous applications in coding theory, communication networks, optimal circuits layouts and crystallography. For a dynamic survey on graph labeling we refer to Gallian [3]. We provide a brief summary of results which will be useful for the present investigations.

Definition 1.1. If the vertices of the graph are assigned values subject to certain conditions then it is known as graph labeling.

A detailed study on variety of applications of graph labeling is reported in Bloom [1].

Definition 1.2. Let $G$ be a graph. A mapping $f: V(G) \rightarrow\{0,1\}$ is called binary vertex labeling of $G$ and $f(v)$ is called the label of the vertex $v$ of $G$ under $f$.

Pradhan and Kumar [4] have analyzed the $\mathrm{L}(2,1)$-Labeling on $\alpha$-Product of Graphs. For an edge $e=u v$, the induced edge labeling $f^{*}: E(G) \rightarrow\{0,1\}$ is given by $f^{*}(e)=|f(u)-f(v)|$. Let $v_{f}(0), v_{f}(1)$ be the number of vertices of $G$ having labels 0 and 1 respectively under $f$ while $e_{f}(0), e_{f}(1)$ be the number of edges of 


\section{A.Sugumaran and P.Vishnu Prakash}

$G$ having labels 0 and 1 respectively under $f^{*}$.

Vaidya and Vyas [7] have proved antimagic labeling of some path and cycle related graphs.

Definition 1.3. A binary vertex labeling $f$ of a graph $G$ is called a cordial labeling if $\left|v_{f}(0)-v_{f}(1)\right| \leq 1$ and $\left|e_{f}(0)-e_{f}(1)\right| \leq 1$. A graph $G$ is called cordial if it admits cordial labeling.

The concept of cordial labeling was introduced by Cahit [2]. Thirusangu et al. [6] proved some results on cordial labeling of duplicate graph of ladder graph.

Definition 1.4. A prime cordial labeling of a graph $G$ with vertex set $V(G)$ is a bijection $f: V(G) \rightarrow\{1,2,3, \ldots,|V(G)|\}$ defined by

$$
f(e=u v)=\left\{\begin{array}{c}
1, \text { if } \operatorname{gcd}(f(u), f(v))=1 \\
0, \quad \text { otherwise }
\end{array}\right.
$$

further $\left|e_{f}(0)-e_{f}(1)\right| \leq 1$.

A graph which admits prime cordial labeling is called a prime cordial graph. The concept of prime cordial labeling was introduced by Sundaram et al. [5].

Now let us recall the definition of Theta graph and the graph operations such as duplication, switching, fusion and the path union of a graph.

Definition 1.5. A Theta graph is a block with two non-adjacent vertices of degree 3 and all other vertices of degree 2 is called a Theta graph.

Definition 1.6. Duplication of a vertex $v_{k}$ of a graph $G$ produces a new graph $G_{1}$ by adding a vertex $v_{k^{\prime}}$ with $N\left(v_{k^{\prime}}\right)=N\left(v_{k}\right)$. In other words, a vertex $v_{k^{\prime}}$ is said to be a duplication of $v_{k}$ if all the vertices which are adjacent to $v_{k}$ are now adjacent to $v_{k^{\prime}}$.

Definition 1.7. A vertex switching of a graph $G$ is a graph $G_{v}$ obtained by taking a vertex $v$ of $G$, removing all the edges incident to $v$ and adding edges joining $v$ to every other vertex which are not adjacent to $v$ in $G$.

Definition 1.8. Let $u$ and $v$ be any two distinct vertices of a graph $G$. A new graph $G_{1}$ is constructed by fusing (identifying) two vertices $u$ and $v$ by a single vertex $x$ in $G_{1}$ such that every edge which was incident with either $u$ or $v$ in $G$ now incident with $x$ in $G_{1}$.

Definition 1.9. Let $G_{1}, G_{2}, G_{3}, \ldots G_{n}, n \geq 2$ be $n$ copies of a fixed graph $G$. The graph obtained by adding an edge between $G_{i}$ and $G_{i}+1$ for $i=1,2, \ldots n-1$ is called the path union of $G$. 


\section{Prime Cordial Labeling for Theta Graph}

\section{Main results}

Theorem 2.1. The Theta graph $T_{\alpha}$ is not a prime cordial graph.

Proof: If $v_{0}, v_{1}, v_{2}, \ldots, v_{6}$ are the vertices of the Theta graph $T \alpha$ with centre $v_{0}$ and $E(T \alpha)=\left\{v_{i} v_{i}+1 / 1 \leq i \leq 5\right\} \bigcup\left\{v_{0} v_{1}, v_{0} v_{4}, v_{1} v_{6}\right\}$, then $\left|V\left(T_{\alpha}\right)\right|=7$ and $\left|E\left(T_{\alpha}\right)\right|=8$.

We define vertex labeling $f: V\left(T_{\alpha}\right) \rightarrow\{1,2,3, \ldots, 7\}$ as follows.

For the graph $T_{\alpha}$ the possible pairs of labels of adjacent vertices are $(1,2),(1,3),(1,4),(1,5),(1,6),(1,7),(2,3),(2,4),(2,5),(2,6),(2,7)$, $(3,4),(3,5),(3,6),(3,7),(4,5),(4,6),(4,7),(5,6),(5,7),(6,7)$.

Out of these pairs, only the pairs $(2,4),(2,6),(3,6)$ and $(4,6)$ yields the edge label value as 0 and the remaining possible labeling of pairs yields the edge label value as 1. But any labeling of vertices in $T_{\alpha}$ must contain at most any three pairs only from $(2,4),(2,6),(3,6)$ and $(4,6)$. This implies that $e_{f}(0) \leq 3$ and so $e_{f}(1) \geq 5$.

Then $\left|e_{f}(0)-e_{f}(1)\right| \geq 2$.

Therefore, $T_{\alpha}$ is not a prime cordial graph.

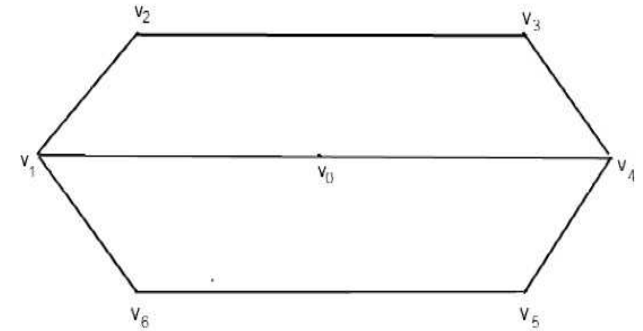

Figure 1: Theta graph

Theorem 2.2. The duplication of any vertex of degree 3 in the Theta graph $T_{\alpha}$ is a prime cordial graph.

Proof: If $v_{0}, v_{1}, v_{2}, \ldots, v_{6}$ are the vertices of the Theta graph $T \alpha$ with centre $v_{0}$ and $E(T \alpha)=\left\{v_{i} v_{i}+1 / 1 \leq i \leq 5\right\} \bigcup\left\{v_{0} v_{1}, v_{0} v_{4}, v_{1} v_{6}\right\}$, then $\left|V\left(T_{\alpha}\right)\right|=7$ and $\left|E\left(T_{\alpha}\right)\right|=8$.

Let $G_{i}$ be a graph obtained from $T \alpha$ after duplication vertex of the vertex $v_{i}$ of degree 3 in $T_{\alpha}$ and $v_{i^{\prime}}$ be the duplication vertex of the vertex $v_{i}$ of degree 3 . In $T_{\alpha}$, only two vertices are of degree 3 . Let it be $v_{1}$ and $v_{4}$.

Clearly $\left|V\left(G_{i}\right)\right|=8$. We define vertex labeling $f: V\left(G_{i}\right) \rightarrow\{1,2,3, \ldots, 8\}$ as follows.

Let $f\left(v_{0}\right)=4$.

Case (i): Duplication of the vertex $v_{4}$.

We define $f\left(v_{4^{\prime}}\right)=8$ where $v_{4^{\prime}}$ is the duplicating vertex of $v_{4}$. 
A.Sugumaran and P.Vishnu Prakash

Further, $f\left(v_{1}\right)=5, f\left(v_{2}\right)=1, f\left(v_{3}\right)=3, f\left(v_{4}\right)=6, f\left(v_{5}\right)=2, f\left(v_{6}\right)=7$.

Case (ii): Duplication of the vertex $v_{1}$.

We define $f\left(v_{1^{\prime}}\right)=8$ where $v_{1^{\prime}}$ is the duplicating vertex of $v_{1}$.

Further, $f\left(v_{1}\right)=6, f\left(v_{2}\right)=3, f\left(v_{3}\right)=1, f\left(v_{4}\right)=5, f\left(v_{5}\right)=7, f\left(v_{6}\right)=2$.

In view of the labeling pattern defined above we have $e_{f}(0)=5, e_{f}(1)=6$.

Thus in both cases we have $\left|e_{f}(0)-e_{f}(1)\right| \leq 1$.

Hence the graph obtained by the duplication of any vertex $v_{i}$ of degree 3 in the Theta graph $T_{\alpha}$ is a prime cordial graph.

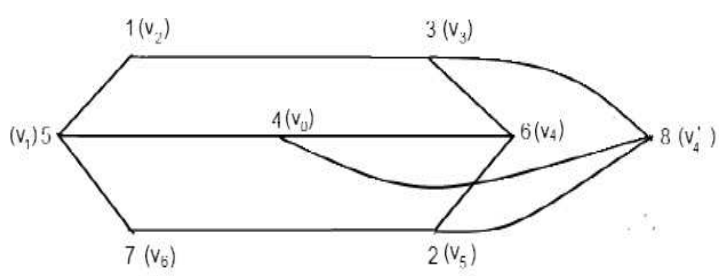

Figure 2: The duplication of the vertex $v_{4}$ in $T_{\alpha}$ is a prime cordial graph

Theorem 2.3. The switching of any vertex of degree 3 in the Theta graph $T_{\alpha}$ is a prime cordial graph.

Proof: If $v_{0}, v_{1}, v_{2}, \ldots, v_{6}$ are the vertices of the Theta graph $T \alpha$ with centre $v_{0}$ and $E(T \alpha)=\left\{v_{i} v_{i}+1 / 1 \leq i \leq 5\right\} \bigcup\left\{v_{0} v_{1}, v_{0} v_{4}, v_{1} v_{6}\right\}$, then $\left|V\left(T_{\alpha}\right)\right|=7$ and $\left|E\left(T_{\alpha}\right)\right|=8$.

Let $G_{s}$ be the graph obtained from $T \alpha$ after switching the vertex $v_{i}$ of degree 3. In $T_{\alpha}$, only two vertices are of degree 3 . Let it be $v_{1}$ and $v_{4}$. Clearly $\left|V\left(G_{s}\right)\right|=7$. We define vertex labeling $f: V\left(G_{s}\right) \rightarrow\{1,2,3, \ldots, 8\}$ as follows.

Let $f\left(v_{0}\right)=3$.

Case (i): switching of the vertex $v_{1}$

We define, $f\left(v_{1}\right)=2, f\left(v_{2}\right)=1, f\left(v_{3}\right)=4, f\left(v_{4}\right)=6, f\left(v_{5}\right)=5, f\left(v_{6}\right)=7$.

Case (ii): switching of the vertex $v_{4}$

We define, $f\left(v_{1}\right)=6, f\left(v_{2}\right)=2, f\left(v_{3}\right)=1, f\left(v_{4}\right)=4, f\left(v_{5}\right)=5, f\left(v_{6}\right)=7$.

In view of the labeling pattern defined above we have $e_{f}(0)=e_{f}(1)=4$.

Thus in both cases we have $\left|e_{f}(0)-e_{f}(1)\right| \leq 1$.

Hence the graph $G_{s}$ admits prime cordial graph. 
Prime Cordial Labeling for Theta Graph

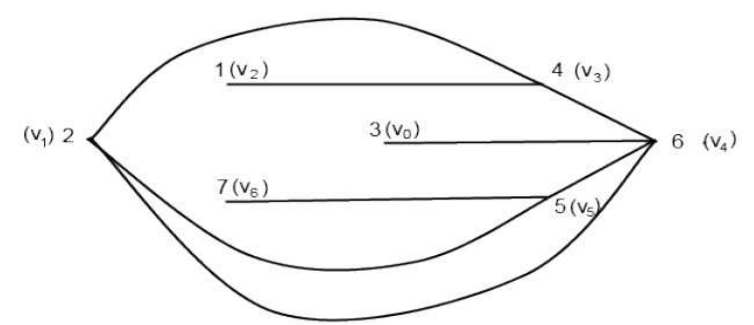

Figure 3: The switching of the vertex $v_{1}$ in $T_{\alpha}$ is a prime cordial graph

Theorem 2.4. The fusion of any two vertices in the Theta graph $T_{\alpha}$ is a prime cordial graph.

Proof: If $v_{0}, v_{1}, v_{2}, \ldots, v_{7}$ be the vertices of the Theta graph $T \alpha$ with centre $v_{4}$ $E(T \alpha)=\left\{v_{i} v_{i}+1 / 1 \leq i \leq 6\right\}$, then $\left|V\left(T_{\alpha}\right)\right|=7$ and $\left|E\left(T_{\alpha}\right)\right|=8$.

Let $G$ be a graph obtained by fusion of any two vertices in $T_{\alpha}$. Then $|V(G)|=6$ and $|E(G)|=7$. We define vertex labeling $f: V(G) \rightarrow\{1,2,3, \ldots,|V(G)|\}$ as follows.

For the graph $G$ the possible pairs of labels of adjacent vertices are $(1,2),(1,3),(1,4),(1,5),(1,6),(2,3),(2,4),(2,5),(2,6),(3,4),(3,5),(3,6),(4,5),(4,6)$, $(5,6)$.

Out of these pairs, only the pairs $(2,4),(2,6),(3,6)$ and $(4,6)$ yields the edge label value as 0 and the remaining possible labeling of pairs yields the edge label value as 1 . We choose the labeling of vertices in $G$ contains any three pairs only from $(2,4),(2,6),(3,6)$ and $(4,6)$.

In view of the labeling pattern defined above we have $e_{f}(0)=3, e_{f}(1)=4$.

Then $\left|e_{f}(0)-e_{f}(1)\right| \leq 1$.

Hence the graph $G$ admits prime cordial graph.

Lemma 2.5. The graph $G$ obtained by path union of two copies of theta graph $T_{\alpha}$ is a prime cordial graph.

Proof: Let $G$ be the graph obtained by path union of two copies of Theta graph $T_{\alpha}$ and $T_{\alpha^{\prime}}$ respectively.

Let $u_{1}, u_{2} \ldots, u_{7}$ be the vertices of first copy $T_{\alpha}$ and $v_{1}, v_{2}, \ldots, v_{7}$ be the vertices of second copy $T_{\alpha^{\prime}}$.

$V(G)=V\left(T_{\alpha}\right) \bigcup V\left(T_{\alpha^{\prime}}\right)$ and $E(G)=E\left(T_{\alpha}\right) \bigcup E\left(T_{\alpha^{\prime}}\right) \bigcup\left\{u_{k} v_{k}\right\}$.

Then $|V(G)|=14$ and $|E(G)|=17$.

We define vertex labeling $f: V\left(G_{s}\right) \rightarrow\{1,2,3, \ldots,|V(G)|\}$ such that

$$
f\left(u_{i}\right)=2 i \text { for } 1 \leq i \leq 7
$$


$f\left(v_{i}\right)=2 i-1$ for $1 \leq i \leq 7$

This implies that $e_{f}(0)=8$ in $T_{\alpha}, e_{f}(1)=8$ in $T_{\alpha^{\prime}}$ and the edge connecting $T_{\alpha}$ and $T_{\alpha^{\prime}}$ gets a label 1 .

In view of the labeling pattern defined above we have $e_{f}(0)=8, e_{f}(1)=9$.

Thus we have $\left|e_{f}(0)-e_{f}(1)\right| \leq 1$.

Hence $G$ is a prime cordial graph.

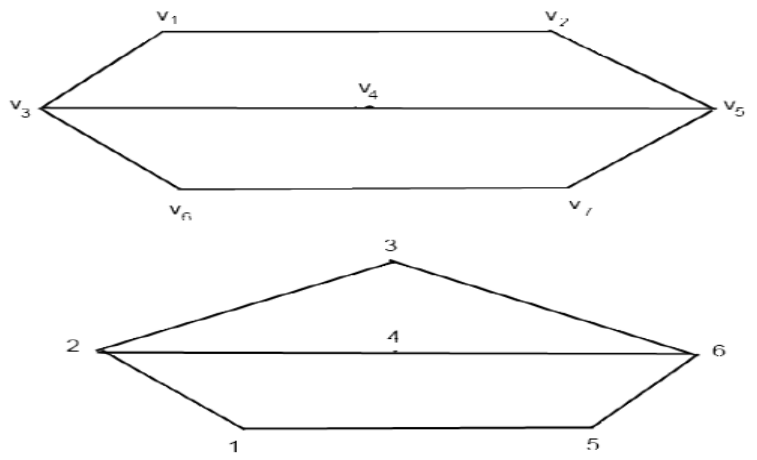

Figure 4: The fusion of the vertex $v_{1}$ and $v_{3}$ in $T_{\alpha}$ is a prime cordial graph

We extend this result to the path of any arbitrary length connecting two copies of Theta graph in the following theorem.

Theorem 2.6. The graph $G$ obtained by joining two copies of theta graph by a path of arbitrary length is prime cordial.

Proof: Let $G$ be the graph obtained by joining two copies of Theta graph by a path $P_{k}$, where $k$ is arbitrary.

Let $u_{1}, u_{2} \ldots, u_{7}$ be the vertices of first copy of Theta graph, $w_{1}, w_{2}, \ldots, w_{7}$ be the vertices of second copy of Theta graph and $v_{1}, v_{2}, \ldots, v_{k}$ be the vertices of path $P_{k}$ and we choose $v_{1}=u_{1}$ and $v_{k}=w_{1}$

Then $|V(G)|=14+k-2$ and $|E(G)|=16+k-1$.

To define vertex labeling $f: V(G) \rightarrow\{1,2,3, \ldots,|V(G)|\}$, we consider the following two cases.

Case 1: $k$ is odd.

Let $k=2 t+1, t \in N$.

We define, $f\left(v_{1}\right)=f\left(u_{2}\right)=3$ and $f\left(v_{k}\right)=f\left(w_{1}\right)$.

Further,

$$
f\left(u_{i}\right)=2 i-1 \text { if } 1 \leq i \leq 7
$$


Prime Cordial Labeling for Theta Graph

$$
f\left(v_{i}\right)=\left\{\begin{aligned}
11+2 i, & \text { if } 2 \leq i \leq t+1 \\
2\{i-(t+1)\}, & \text { if } t+2 \leq i \leq 2 t+1
\end{aligned}\right.
$$

and $f\left(w_{i}\right)=2 t+2(i-1)$ if $1 \leq i \leq 7$.

In view of the labeling pattern defined above we have $e_{f}(0)=e_{f}(1)=\frac{15+k}{2}$.

Thus we have $\left|e_{f}(0)-e_{f}(1)\right| \leq 1$.

Case 2: $k$ is even.

Let $k=2 t+1, t \in N$.

We define, $f\left(v_{1}\right)=f\left(u_{2}\right)=3$ and $f\left(v_{k}\right)=f\left(w_{1}\right)$.

Further,

$$
\begin{aligned}
f\left(u_{i}\right)=2 i-1 & \text { if } 1 \leq i \leq 7 \\
f\left(v_{i}\right) & =\left\{\begin{array}{c}
11+2 i, \quad \text { if } 2 \leq i \leq t \\
2\{i-t\}, \quad \text { if } t+1 \leq i \leq 2 t
\end{array}\right.
\end{aligned}
$$

and $f\left(w_{i}\right)=2 t+2(i-1)$ if $1 \leq i \leq 7$.

In view of the labeling pattern defined above we have when $k=2$, $e_{f}(0)=8, e_{f}(1)=9$.

Also, $e_{f}(0)=8+\frac{k}{2}$ ande $_{f}(1)=7+\frac{k}{2}$ for $k=4,6, \ldots$.

Thus we have $\left|e_{f}(0)-e_{f}(1)\right| \leq 1$.

Hence $G$ is a prime cordial graph.

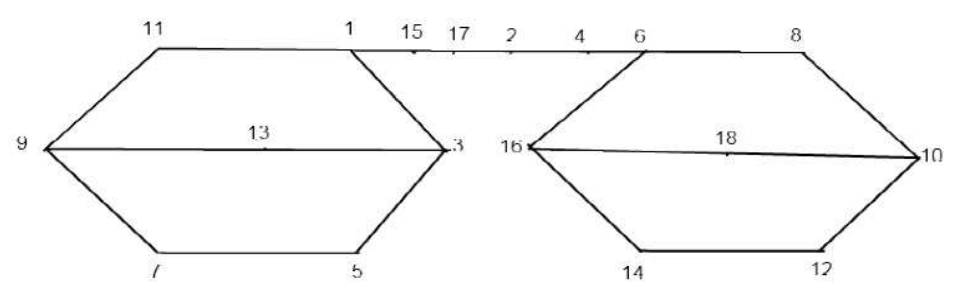

Figure 5: Prime cordial labeling of graph obtained by joining 2 copies of $T_{\alpha}$ by path $P_{6}$

\section{Concluding remarks}

In this paper, we investigated the prime cordial labeling of theta graph. We also proved that the prime cordial labeling in the context of some graph operations namely duplication, witching, fusion, path union and the graph obtained by joining two copies of Theta graph by a path of arbitrary length.

\section{REFERENCES}

1. G.S.Bloom and S.W.Golomb, Applications of numbered undirected graphs, Proc of IEEE, 65(4) (1977) 562-570.

2. I.Cahit, Cordial Graphs: A weaker version of graceful and harmonious Graphs, Ars 


\section{A.Sugumaran and P.Vishnu Prakash}

Combinatoria, 23 (1987) 201-207.

3. J.A.Gallian, A dyanamic survey of graph labeling, Electronic Journal of Combinatorics, 16 (2016) \# DS6.

4. P.Pradhan and Kamesh Kumar, The L(2,1)-labeling on $\alpha$-product of graphs, Annals of Pure and Applied Mathematics, 10(1) (2016) 29-39.

5. M.Sundaram, R.Ponraj and S.Somasundaram, Prime cordial labeling of graphs, Journal of Indian Academy of Mathematics, 27 (2005) 373-390.

6. K.Thirusangu, P.P.Ulaganathan and P.Vijaya Kumar, Some cordial labeling of duplicate graph of ladder graph, Annals of Pure and Applied Mathematics, 8(2) (2014) 43-50.

7. S.K.Vaidya and N.B.Vyas, Antimagic labeling of some path and cycle related graphs, Annals of Pure and Applied Mathematics, 3(2) (2013) 119-128. 